\title{
High Voltage Circuit Breakers: SF6 vs. Vacuum
}

\author{
A. Iturregi ${ }^{1}$, E. Torres $^{2}$, I. Zamora $^{3}$, O. Abarrategui ${ }^{4}$ \\ ${ }^{1}$ Department of Electrical Engineering \\ UPV-EHU \\ Colina Beurko s/n, 48901 Barakaldo (Spain) \\ Phone/Fax number: +34 94601 4979/7800, e-mail: araitz.iturregi@ehu.es ${ }^{1}$, esther.torresi@ehu.es ${ }^{2}$, \\ inmaculada.zamora@ehu.es ${ }^{3}$, oihane.abarrategui@ehu.es ${ }^{4}$
}

\begin{abstract}
As important as finding new renewable and environmentally friendly sources of energy is the fact of transmitting and distributing this energy in the safest and most efficient way. In this field, High Voltage Circuit Breakers (HV $\mathrm{CB})$, designed for operation of the electric network under normal conditions as well as for the interruption of faulted conditions, have played an important role in power systems over 100 years. However, research, development and improvement of the design of CBs have not stopped in our nowadays expanding grid.
\end{abstract}

The aim of this paper is to make a review of the existing different types of $\mathrm{HV} \mathrm{CB}$, comparing the most used one, SF6 $\mathrm{CB}$, with the Vacuum $\mathrm{CB}$, which has been developed and recently applied for $\mathrm{HV}$, and could become the future environmental friendly HV CB.

\section{Key words}

Circuit Breaker (CB), Vacuum Circuit Breaker (VCB), Sulfurhexaflouride (SF6), Electric arc, Vacuum Arc.

\section{Introduction}

A Circuit Breaker is a switching device which can open or close a circuit in a small fraction of second. This is achieved due to its separable contacts. The closing and opening of the circuit allows to establish or to interrupt the circulation of current through the circuit under usual or unusual working conditions, such as short circuits.

The interruption process of the current in a $\mathrm{CB}$, begins when the movable contacts start to separate. As a consequence, the contact area is reduced and the current density gets larger, until the energy causes the metal to begin vaporizing and an arc appears. In spite of the existence of a physical separation of the switching contacts, the established arc makes possible that the current continues flowing (Figure 1). The interruption of the circulating current will be achieved when the interrupting medium gets to turn the carrying arc plasma into an isolating medium. ([1]-[4])

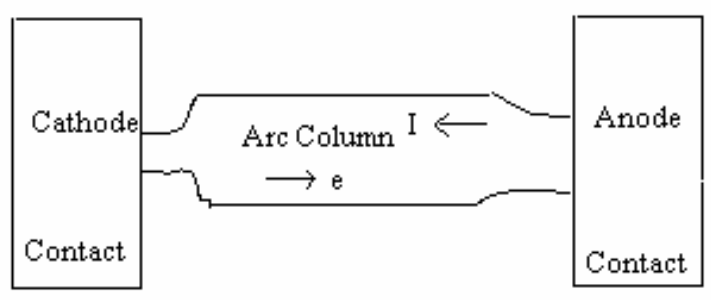

Figure 1. Electric arc structure.

In the beginning of the arc phenomena the main source of charged particles is the electrode vapour. However, as the contact separation increases the ionization degree of the arc column is also influenced by the characteristics of the surrounding medium, except for Vacuum CBs.

Attending to the medium and the method used for the interruption of the current, CBs can be grouped in the following types:

- Air Magnetic Circuit Breakers

- Air Blast Circuit Breakers

- Oil Circuit Breakers

- Sulfurhexaflouride (SF6) Circuit Breakers

- Vacuum Circuit Breakers

Oil circuit breakers were the first $\mathrm{CB}$ type in the grid, due to its ability to interrupt large currents. The interruption process is based on the creation of hydrogen and acetylene gas bubble, as the oil decomposes as a result of the arc established between the switching contacts. The disadvantages of using oil as quenching media in circuit breakers, as flammability and a high maintenance cost, forced to search for different mediums of quenching. Air Blast and Magnetic Air circuit breakers were developed but did not sustain in the market due to some disadvantages, as the fact that they are bulky and cumbersome. In the middle of the century, and being 
considered as the new generation of CBs, SF6 and Vacuum CBs appeared.

SF6 CBs started quickly to replace oil and air CBs for $\mathrm{HV}$ applications, as most SF6 properties are superior to other interrupting mediums, such as its high dielectric strength or higher thermal conductivity. In contrast, Vacuum CBs started to spread in the Medium Voltage level up to rated voltages of around $36 \mathrm{kV}$. ([1], [5])

Even though many CBs from the first generation, with oil or air as quenching medium, are still working, SF6 CB is undoubtedly the most common $\mathrm{CB}$ used nowadays for HV applications worldwide. But, it also has its limitations and disadvantages. Vacuum switching, widely used for medium voltage levels $(5-38 \mathrm{kV})$, has emerged as an alternative for high voltage applications due to its environmental friendliness. Latest research on contact material composition, magnetic field application, insulation, multi-gap or long gap technology has lead to the development of various prototypes for higher voltage levels. Following, both types, SF6 and vacuum are analysed in detail.

\section{SF6 CBs}

In SF6 circuit-breakers, the current continues flowing after contact separation through an arc plasma of ionized SF6. The circuit breaker is designed to direct a constant gas flow to the arc that extracts heat from the arc and so allows achieving its extinction at current zero. The gas flow de-ionises also the contact gap and restablishes the required dielectric strength to prevent an arc re-strike.

The direction of the gas flow, i.e., whether it is parallel to or across the axis of the arc, has a decisive influence on the efficiency of the arc interruption process. Research has shown that an axial gas flow creates a turbulence which causes an intensive and continuous interaction between the gas and the plasma as the current approaches zero. Cross-gas-flow cooling of the arc is generally achieved, in practice, by making the arc to move in the stationary gas. However, this interruption process can lead to arc instability and resulting great fluctuations in the interrupting capability of the circuit breaker.

In order to achieve an axial gas flow, a differential pressure must be created along the arc. The first generation of SF6 CBs used the two-pressure principle of the air-blast CB. A certain quantity of gas was kept stored at high pressure and released into the arcing chamber. In later designs, the differential pressure was created by a piston attached to the moving contacts, which compresses the gas in a small cylinder as the contact opens. Some disadvantages are the relatively powerful operating mechanism required by this puffer system and the cost of this operating mechanism.

Consequently, new developments were aimed at reducing or eliminating this additional cost factor. These developments concentrated on employing the arc energy itself to create directly the differential pressure needed.
This research led to the development of the selfpressuring circuit-breaker in which the overpressure is created by using the arc energy to heat the gas under controlled conditions. During the initial stages of development, an auxiliary piston was included in the interrupting mechanism, in order to ensure the satisfactory breaking of small currents. Subsequent improvements in this technology have eliminated this requirement and in the latest designs, the operating mechanism must only provide the energy needed to move the contacts.

Parallel to the development of the self-pressuring design, the rotating arc SF6 interrupter appeared. In this design a coil sets the arc in rotation while the quenching medium remains stationary. The relative movement between the arc and the gas is no longer axial but radial, i.e., it is a cross-flow mechanism. The operating energy required by circuit breakers of this design is also minimal [1].

\section{Vacuum CBs}

\section{A. General features}

When the contacts separate in a VCB, the current flowing trough those electrodes initiates a metal vapour arc discharge in the contacts called the vacuum arc. The vacuum arc, once established, flows through the plasma in a self sustained way until the next current zero. The arc is then extinguished and the conductive metal vapour condenses on the metal surfaces, within a matter of micro seconds. As a result, the dielectric strength in the breaker builds up very rapidly. Figure 2 shows the internal components of a typical vacuum interrupter.

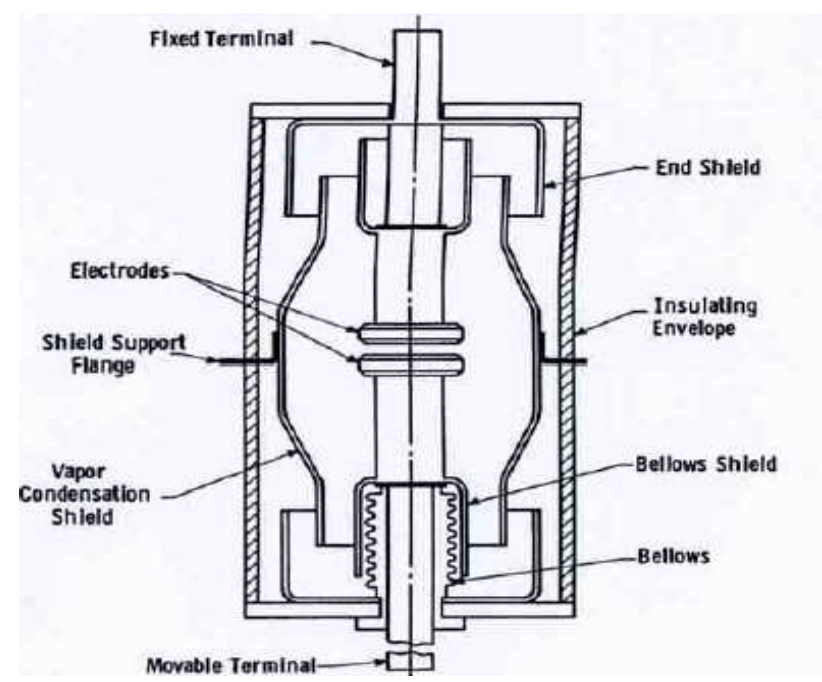

Figure 2. Vacuum interrupter structure [3].

\section{B. MV application}

Medium voltage is where VCB has found its widest application field and is widely used in the range of 5 to $38 \mathrm{kV}[1]$.

Vacuum arcs have more than one appearance, as there are different modes of vacuum arcs. The mode of the vacuum arc depends on the current level and the size of the 
contacts. At lower currents, the arc assumes the diffuse mode. The characteristics of diffuse arcs are that there are one or more cathode spots moving constantly over the contact surface. These spots repel each other and they have a finite and variable lifetime. While some cathode spots are extinguished, some others split, creating new ones. As the current increases, the arc changes its appearance. All the plasma is focused on a small area of the electrode, about $1 \mathrm{~cm}^{2}$, so instead of multiples moving cathode spots the appearance that the arc takes is a constricted mode.

The current at which the transition from diffuse to constricted mode takes place, depends on the size of the contact and its material but it is usually between 10 and $15 \mathrm{kA}$. The constricted mode of the arc has nothing but disadvantages, as the interruption of the current is much more difficult, and it produces severe erosion on contacts. Besides, a constricted arc that remains on one spot for too long can thermically over stress the contacts to such a degree that the deionization of the contact zone at zero current can no longer be guaranteed ([1], [6]).

To overcome this problem the arc root must be moved over the contact surface and in order to achieve this, contacts are appropriately shaped so that the current flowing through them results in a radial magnetic field. This radial field, also known as transverse field, causes the arc root to rotate rapidly around the contact, resulting in a uniform heat distribution over its surface. The contacts of this type are called radial magnetic field or spiral electrodes (Figure 3).

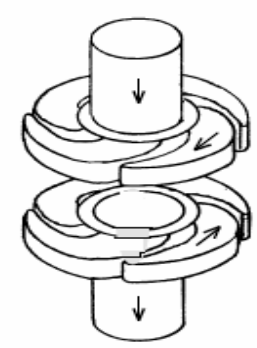

Figure 3. Radial magnetic field electrodes [7]

The spiral electrodes are able to interrupt a current of $30 \mathrm{kA}$ with electrodes of $100 \mathrm{~mm}$ of diameter. As it is shown in Figure 4, the electrodes size becomes too large for high current interruptions.

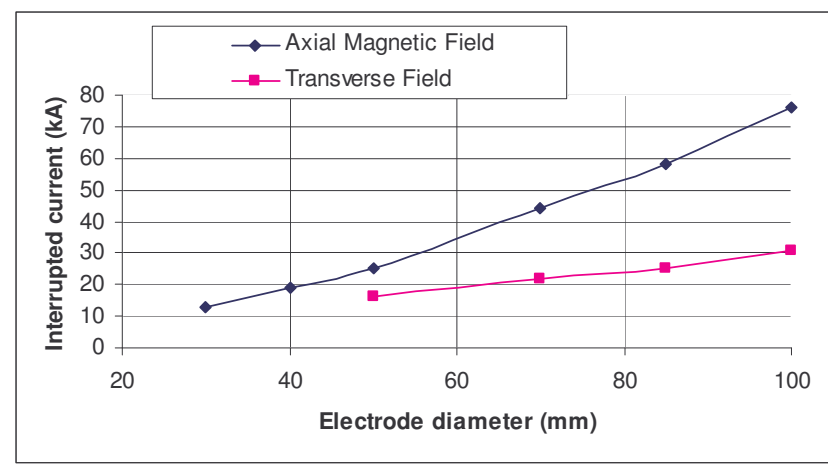

Figure 4. Interruption capability for vacuum interrupters as function of electrode diameter and magnetic field type.

\section{HV application}

At high voltage, the vacuum arc adopts a constricted form, which makes more difficult the arc quenching at current zero. But the problem of switching the arc from constricted to diffuse, in $\mathrm{HV}$ applications, can not be achieved by subjecting the arc to a radial magnetic field, as it is solved for MV. This is so because the current would not be interrupted unless the size of the contacts were large enough. And such big electrodes would make the VCB bulky and not so competitive [1].

In contrast, for high voltage applications an axial magnetic field is applied (Figure 4). The axial magnetic field can be provided by leading the arc current through a coil, suitably arranged outside the vacuum chamber. Alternatively, the field can be provided by a suitable contact design. Such contacts are called axial magnetic field electrodes (Figure 5). With the higher magnetic field strength, the radial arc distribution is more homogenized. This way the arc energy is reduced.

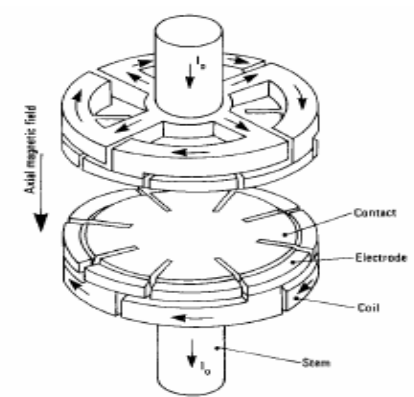

Figure 5. Axial magnetic field electrodes [8]

Another problem in these applications, HV VCB, is related to the high-voltage capability of a single gap between electrodes. The breakdown characteristics have very high dependence of electrode area, and the dielectric strength with the contact gap. On this way, in vacuum breakdown, the breakdown voltage is proportional to the square root of the gap length [9].

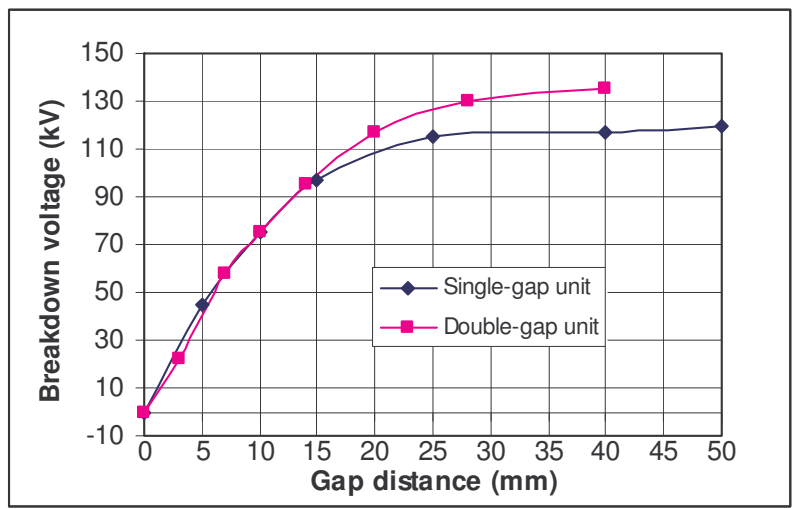

Figure 6. Double and single gap interrupters breakdown voltage capacity as a function of contact separation.

In Figure 6 it can be observed the behaviour of a singlegap interrupter. Until $10 \mathrm{~mm}$ the breakdown voltage is linear to the distance, but for larger contact distances the curve describes an inflected characteristic, not being 
linear any more. Thus, a longer gap is necessary for the vacuum interruption. More or less $50 \mathrm{~mm}$ of separation is used for single-break interrupters, what makes the $\mathrm{CB}$ bigger. This technology, with higher current and longer contact spacing, leads to the problem of the control of the arc. It appears a high arc voltage noise, which indicates that the vacuum arc is unstable in a long gap [11].

Another solution for the same problem is the multi-gap technology, that consists in series-connected interrupters (Figure 7). In Figure 6 the behaviour of both configurations, single and double-gap, is shown. For example, two series connected gaps of $20 \mathrm{~mm}$ each can substitute a single long gap of $50 \mathrm{~mm}$.
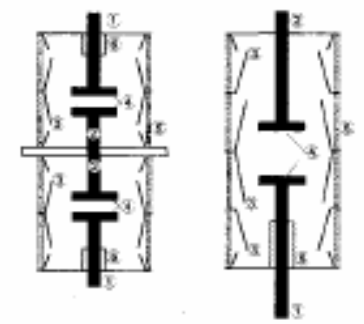

Figure 7. Double gap series-connected interrupter on the left and single long gap on the right [9].

Other aspect to take into account is the melting and the erosion in the contacts, as the higher the voltage, the higher the erosion and melting. The construction material of the electrode is the essential factor for determining the erosion and melting. As a result of the development of different types of material combination for vacuum electrodes, as for example $\mathrm{Cu}, \mathrm{Cu}-\mathrm{Bi}$ or $\mathrm{Cu}-\mathrm{Cr}$, an oxygen free copper chromium alloy, $\mathrm{Cu}-\mathrm{Cr}$, seems to be the best one until now (Figure 8). In this alloy, chromium is distributed through copper in the form of fine grains. This material combines good arc extinguishing characteristics with a reduced tendency to contact welding. Using $\mathrm{Cu}$, the melting in the large current range is limited, and using $\mathrm{Cr}$ a higher withstand voltage is achieved. Also a small reignition probability is obtained [8].

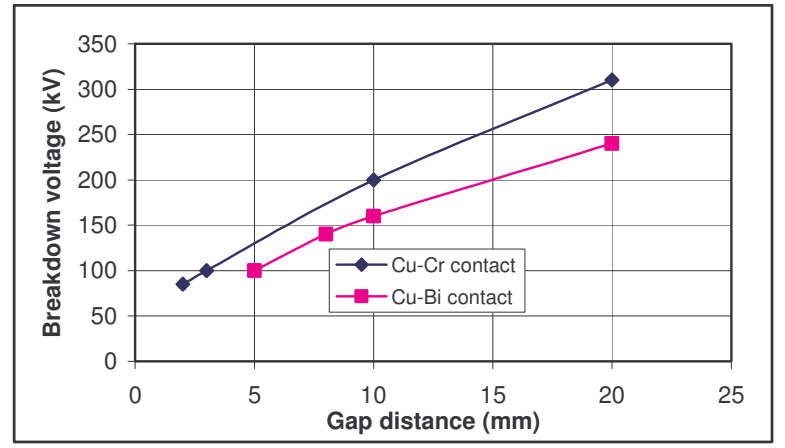

Figure 8. Breakdown voltage of $\mathrm{Cu}-\mathrm{Cr}$ and $\mathrm{Cu}-\mathrm{Bi}$ contacts as a function of contact separation.

\section{VCB prototypes developed for $\mathrm{HV}$}

In this section some prototypes of HV VCB developed from different companies and researchers are presented:
- In 1979 a $168 \mathrm{kV} / 31,5 \mathrm{kA}$ VCB was developed in Japan, shown in Figure 9. The $\mathrm{CB}$ was a double break $\mathrm{CB}$, and even if the breakdown voltage was high, the $\mathrm{CB}$ was too expensive and large [13].

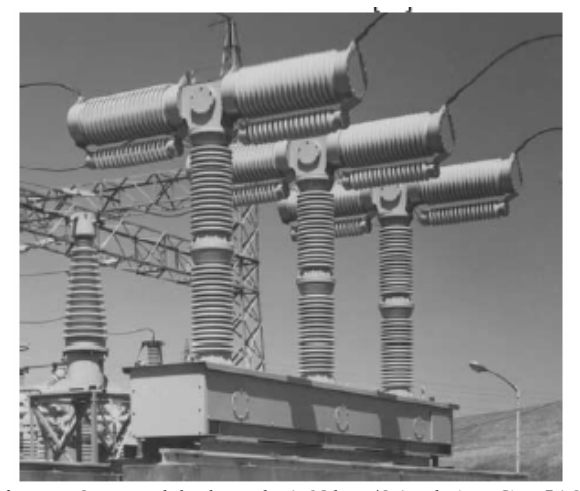

Figure 9. Double break 168kV/31,5kAVCB [13]

- Another VCB developed by Japan AE Power System Corporation in 2002, improved the previous design (Figure 10). The rated voltage of this $\mathrm{CB}$ is $145 \mathrm{kV}$, the short circuit current $40 \mathrm{kA}$ and the rated current $2 \mathrm{kA}$. The interrupter used is a single long-gap, one break vacuum interrupter (Figure 11), where axial magnetic field electrodes of $\mathrm{Cu}-\mathrm{Cr}$ are used (Figure 5). The achievements were a magnetic axial field distribution on electrode structure, electrode erosion reduction and temperature analysis, as well as, a simpler structure and a lower cost ([7], [10], [13]).



Figure 10. Single-gap 145kV/40kA/2kA VCB [10].

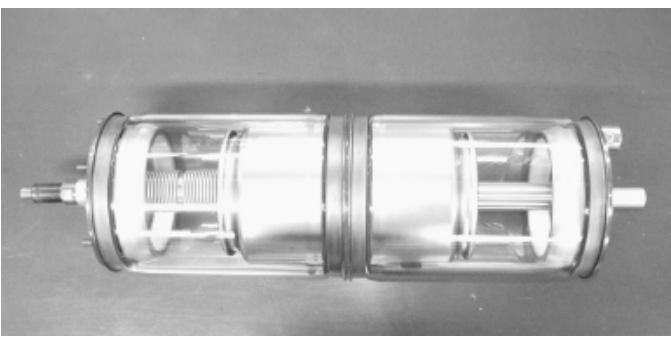

Figure $11.145 \mathrm{kV}$ vacuum interrupter, only $700 \mathrm{~mm}$ length and $200 \mathrm{~mm}$ diameter [7].

- In 1989, a Chinese researchers' team presented a $126 \mathrm{kV} / 31,5 \mathrm{kA} / 1,25 \mathrm{kA}$ double-break VCB. This CB was achieved using two vacuum interrupters of $72.5 \mathrm{kV}$ 
connected in series (double-break). The contact gap was $40 \mathrm{~mm}$ length for each interrupter, axial magnetic field type electrodes were used and external insulation of SF6 was also applied. The CB had only $2650 \mathrm{~mm}$ height (Figure 12) [11].

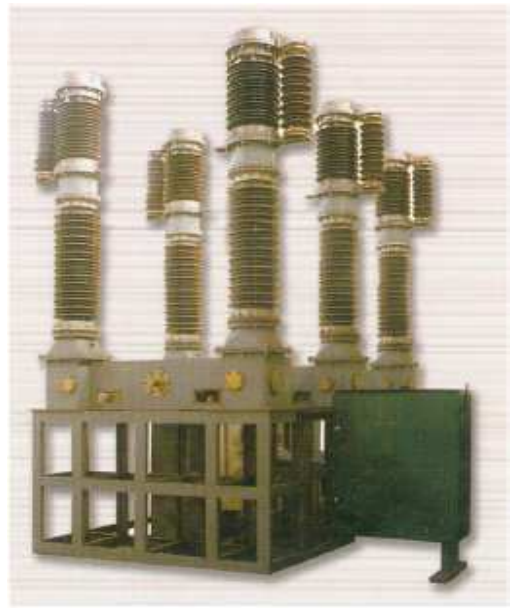

Figure 12. Double break $126 \mathrm{kV} / 31,5 \mathrm{kA} / 1,25 \mathrm{kA}$ VCB (height $2650 \mathrm{~mm})$ [11]

- In 2003 a $126 \mathrm{kV} / 40 \mathrm{kA} / 2 \mathrm{kA}$ single-break VCB was developed (Figure 13 and 14). The interrupter had a diameter of $100 \mathrm{~mm}$ and the contact gap length needed was $60 \mathrm{~mm}$. The contact gap was longer than in the previous case but just one break was needed. The electrodes used were of the axial magnetic fields type [11].

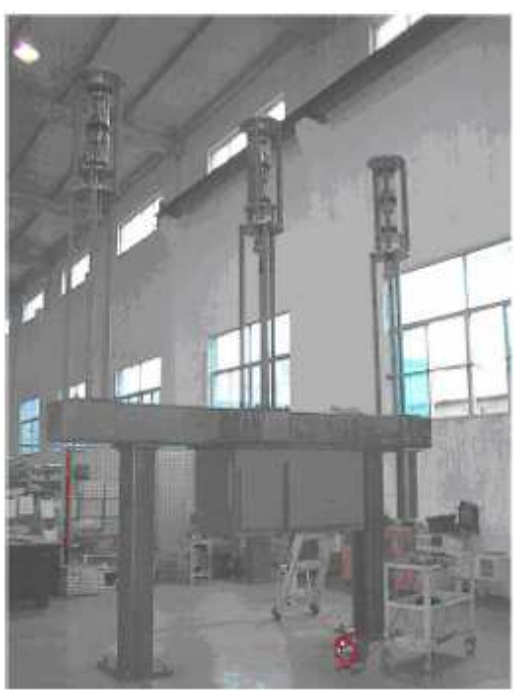

Figure 13. Single-break 126kV/40kA/2kA VCB [11]

- Finally, in 2006, the first prototype of a $252 \mathrm{kV} / 40 \mathrm{kA}$ VCB was achieved [11] (Figure 14). This CB is a singlebreak prototype with axial magnetic field type electrodes whose diameter is $140 \mathrm{~mm}$, being the contact gap length $80 \mathrm{~mm}[11]$.

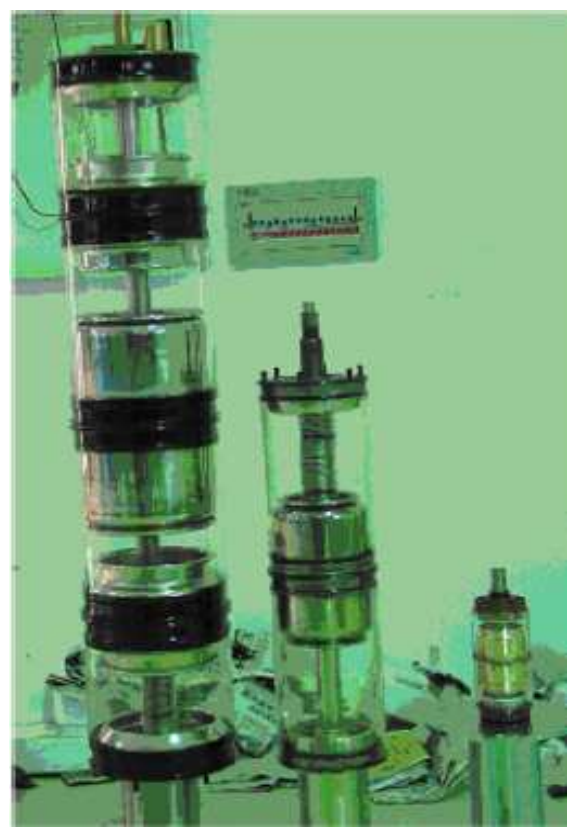

Figure 14. Vacuum interrupters of $252 \mathrm{kV}, 126 \mathrm{kV}$, and $12 \mathrm{kV}$

[11]

Additionally, more prototypes can be found in references [13], [14] and [15].

\section{Comparison between SF6 and Vacuum CBs}

In this section, SF6 and vacuum circuit breakers are compared considering different factors, as for example the dielectric strength and environmental effect.

According to the dielectric strength, SF6 has better behaviour than vacuum (Figure 15). That is why SF6 has generalized as insulating and as arc quenching medium. The use of SF6 makes possible to reduce the size of electric equipment and so the required space for installation. That is the reason why its most important use, about $50 \%$ of the total, is as dielectric in electric devices, as CBs.

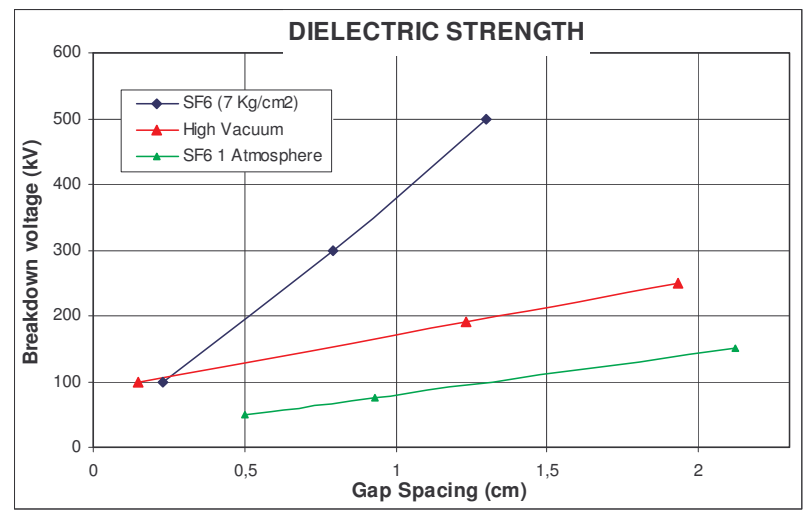

Figure 15. Dielectric strength of SF6 and Vacuum.

Besides, under normal conditions, SF6 is an inert, nonflammable, non-corrosive, odourless and non-toxic gas. However, at temperatures over $1000^{\circ} \mathrm{C}$, SF6 decomposes to gases including $\mathrm{S}_{2} \mathrm{~F}_{10}$ which is highly toxic. Fortunately, the decomposition products recombine abruptly after arc extinction (when the temperature goes 
down). It could be said then, that SF6 would be the perfect quenching medium for a $\mathrm{CB}$ if it was not environmentally harmful. SF6 has been labelled as one of the major global warming gases, since the $3^{\text {rd }}$ Session of the Conference of the Parties to the United Nations Framework Convention on Climate Change. The fact that SF6 is a special global warning contributory gas is based on its stable molecular composition, as it becomes indestructible for a long period of time, over 3200 years.

In Table I a comparison between several gases with regards to their lifetime years and their Global Warming Potential (GWP) is shown.

TABLE I. Global Warming Potential.

\begin{tabular}{|l|l|l|}
\hline Compound & Lifetime Years & GWP \\
\hline $\mathrm{CO}_{2}$ & $50-200$ & 1 \\
\hline $\mathrm{CF}_{4}$ & 50.000 & 6.300 \\
\hline $\mathrm{C}_{2} \mathrm{~F}_{6}$ & 10.000 & 12.500 \\
\hline $\mathrm{SF}_{6}$ & 3.200 & 24.900 \\
\hline $\mathrm{C}_{6} \mathrm{~F}_{14}$ & 3.200 & 6.800 \\
\hline
\end{tabular}

In contrast, as in VCBs the quenching media is vacuum, there is no risk for the environment. In fact, they consist on an ordinary glass container and metallic components, that is, recyclable materials[16].

Vacuum brings its own advantages and disadvantages, which differ from those of SF6. Among the advantages that VCBs present, there can be highlighted that the construction of VCBs is simple and the number of components is small, approximately $50 \%$ less than in a SF6 CB. That leads to a long operation life, with very high number of operating cycles. Besides, the small amount of components and simple construction also brings a compact size and a light weight for VCBs, and consequently an easy maintenance and inspection.

Another advantage of VCBs is the higher dielectric resistance recovery after current zero.

And finally, as it has been mentioned, there is no environmental damage in a VCB, as in the case of SF6 $\mathrm{CBs}$ and neither danger of explosion or fire as there is for oil CBs.

However, a major disadvantage is their cost. SF6 CBs are still cheaper, what does not make a VCB competitive. More work must be done in order to reduce the cost of $\mathrm{VCBs}$ so that they can become an economic alternative to the SF6 technology.

\section{Conclusion}

The non stopping growing electric power grid demands increased performance and reliability, but also sustainability. Thus, it is important to continue developing new $\mathrm{CB}$ technology, more reliable, more capable, less costly, environmentally friendly, recyclable, and safe for humans. Vacuum is a medium with outstanding properties concerning size, amount of components, simplicity, short-circuit current control or dielectric strength recovery. The applications, today still mostly in medium voltage distribution networks, will potentially be widely spread to HV distribution networks, avoiding the environmental impact of SF6. But, it is necessary to introduce changes in the design and the materials used to ensure the proper working of VCB at higher voltage values.

\section{References}

[1] R.D. Garzón, High Voltage Circuit Breakers. Marcel Dekker, Inc. 2002.

[2] C.L. Wadhwa, Electrical Power Systems. John Wiley \& Sons, Inc. 1991.

[3] T.E. Browne, Circuit Interruption. Theory and Techniques. Marcel Dekker, Inc., 1984.

[4] C.H. Flurscheim, Power Circuit Breaker Theory and Design. Peter Peregrinus Ltd, 1982.

[5] J.H Brunke, "Circuit-Breakers: Past, present and future". Electra n'208. June 2003.

[6] A. Greenwood, Vacuum Switchgear. The Institution of Electrical Engineering. 1997.

[7] H. Saitoh, H. Ichikawa, A. Nishijma, Y. Matsui, M. Sakaki, M. Honma, H. Okubo, "Research and development on $145 \mathrm{kV} / 40 \mathrm{kA}$ one break vacuum circuit breaker" IEEE T/D Conference, Yokohama, p1462-1468, 2002.

[8] S. Yanabu, T. Tsutsumi, K. Yokokura, and E. Kaneko "Recent Technical Developments in High-Voltage and HighPower Vacuum Circuit Breakers". IEEE Transaction on plasma science. Vol. $17 \mathrm{n}^{\circ}$ 5. October 1989, pp. 717-723.

[9] S. Giere, H.C. Kärner, H. Knobloch, "Dielectric strength of double and single-break vacuum interrupters", IEEE Transactions on dielectric and electrical insulation, Vol.8 No.1, February 2001, pp 43-47.

[10] Y. Ohki, "Japan AE power systems develops high-voltage, vacuum circuit breakers". IEEE Electrical Insulation Magazine. Vol. $23 n^{\circ} 1$, January/February 2007, pp 48-49.

[11] Z.Liu, J. Wang, S. Xiu, Z. Wang, "Development of HighVoltage Vacuum Circuit Breakers in China". IEEE Transaction on plasma science. Vol.35 n 4, August 2007, pp 856-865.

[12] H. Schellekens, G. Gaudart, "Compact High-Voltage Vacuum Circuit Breaker, a Feasibility Study", IEEE Transactions on dielectric and electrical insulation, Vol.14 No.3, June 2007, pp 613- 619.

[13] M. Homma, M. Sakaki, E. Kaneko, S. Yanabu, "History of Vacuum Circuit Breakers and Recent Developments in Japan". IEEE Transaction on dielectrics and electrical insulation. Vol.13, n ${ }^{\circ} 1$. February 2006, pp. 85-92.

[14] R.B Shores, V.E. Phillips, "High Voltage Vacuum Circuit Breakers" IEEE Transaction on power apparatus and systems. Vol.94, no 5, September/October 1975, pp 1821-1830.

[15] P.G. Slade, R.E. Voshall, P.O.Wayland, A. Bamford, G. McCracken, R. Yeckley, H. Spindle, "The development of a vacuum interrupter retrofit for the upgrading and life extension of $121 \mathrm{kV}-145 \mathrm{kV}$ oil circuit breakers" IEEE Transactions on power delivery, Vol.6 No.3, July 1991, pp 1124-1131.

[16] P. Rollet, J. Micozzi, "SF6 recycling". ftp://ftp.ecn.nl/pub/www/library/conf/ipcc99/m99a7-2.pdf.

[17] M. Budde, A. Horn, F. Körner, M. Kurrat, K. Steinke, "Dielectric Behaviour of Vacuum Circuit-Breakers". www.htee.tu-bs.de/forschung/veroeffentlichungen/ kurrat_paper_dielectric_behaviour_ISH2005.pdf. 